\title{
Un inédit de Buonarroti : la " Réplique à la réponse de l'accusateur national »
}

\section{Stéphanie Roza}

\section{Q OpenEdition \\ 1 Journals}

\section{Édition électronique}

URL : https://journals.openedition.org/ahrf/12535

DOI : $10.4000 /$ ahrf.12535

ISSN : 1952-403X

Éditeur :

Armand Colin, Société des études robespierristes

\section{Édition imprimée}

Date de publication : 1 décembre 2012

Pagination : 213-233

ISBN : 978-2-200-92762-2

ISSN : 0003-4436

\section{Référence électronique}

Stéphanie Roza, « Un inédit de Buonarroti : la « Réplique à la réponse de l'accusateur national » », Annales historiques de la Révolution française [En ligne], 370 | octobre-décembre 2012, mis en ligne le 01 décembre 2015, consulté le 23 avril 2022. URL : http://journals.openedition.org/ahrf/12535 ; DOI : https://doi.org/10.4000/ahrf.12535 


\title{
UN INÉDIT DE BUONARROTI : LA «RÉPLIQUE À LA RÉPONSE DE L'ACCUSATEUR NATIONAL »
}

Stéphanie ROZA

\begin{abstract}
À la fin des années 1920, Gustave Fontaine, maire socialiste de la commune de Coudekerque-Branche, dans le Nord, fait l'acquisition chez un bouquiniste lillois d'un épais cahier manuscrit, anonyme, contenant trois chapitres intitulés respectivement « Défense de Buonarroti », « Discussion particulière à Buonarroti » et enfin «Réplique à la réponse de l'accusateur national ». Ces titres évoquaient sans doute possible le fameux procès, intenté en Haute Cour conformément à la lettre de la constitution de 1795, aux auteurs et supposés complices de la Conspiration des Égaux dite de Babeuf, conspiration dans laquelle Buonarroti avait joué un rôle majeur, pour lequel il avait été condamné à la déportation de 1797 à 1800, et dont il avait été par la suite (en 1828) le premier chroniqueur.

Gustave Fontaine avait toutes les raisons de se passionner pour le document. Maire socialiste, féru d'histoire, porté sur le récit des événements de la Révolution Française, il nourrissait un respect particulier pour Robespierre, tout comme Buonarroti en son temps, dont il était également, selon ses propres mots, un « admirateur».

Bientôt, il entreprit de vastes recherches visant à établir l'authenticité du manuscrit, l'identité de son ou ses auteurs, ainsi que le destin ultérieur de ces lignes manifestement écrites pour être lues à la barre du tribunal. En 1935 il entra en correspondance avec Georges Lefebvre, président de la Société des Études Robespierristes, qui l'encouragea dans ses démarches. Un travail minutieux lui permit de dénombrer trois écritures différentes, dont celle de Buonarroti qui n'apparaît cependant que pour quelques dizaines de retouches ponctuelles. Manifestement, d'autres que lui avaient recopié sa plaidoirie, qui au vu des circonstances du procès ne pouvaient être que des co-accusés. L'historien-maire parvint à établir que les premier et troisième chapitres du document étaient presque assurément de la main
\end{abstract}


de Babeuf ; la deuxième pouvant être soit de celle de Darthé, soit de Germain, deux importants animateurs du mouvement, membres de ce qui se dénommait le «Comité insurrecteur ».

Les deux premières parties de ce manuscrit se retrouvent, au mot près, les retouches de Buonarroti exceptées, dans le compte-rendu sténographié édité aux frais du gouvernement au moment du procès lui-même, en $1797^{1}$. La troisième partie ne s'y trouve pas. Cette absence permet à Gustave Fontaine (et à nous après lui) de conclure que celle-ci n'a finalement pas été prononcée, et donc qu'elle est inédite, malgré l'annonce, faite par Buonarroti dans un prospectus paru à Paris en $1830^{2}$, d'une parution prochaine dont on n'a retrouvé aucune trace.

Cette copie très propre et très lisible des discours, ainsi que les retouches de Buonarroti sur le manuscrit, probablement postérieures à la tenue du discours lui-même puisqu'elles n'apparaissent pas dans le compte-rendu sténographié publié, laissent penser que Buonarroti et ses compagnons d'infortune, projetaient dès l'époque de publier l'ensemble, ce qui ne s'est pas réalisé.

Comment ce texte s'est-il conservé jusqu'à atterrir chez un bouquiniste de Lille ? Nous l'ignorons. Gustave Fontaine poursuit ses recherches dans les fonds d'archives et sa correspondance avec la Société des études robespierristes jusqu'en 1935. Il meurt le 25 février 1937 sans avoir pu achever son travail. En 2011, son petit-fils Pierre Fontaine fait parvenir une copie du manuscrit au secrétariat de la Société, qui le remet à Claude Mazauric. Celui-ci, empêché, le transmet à l'auteure de cette notice aux fins d'en préparer l'édition. Qu'il soit rendu ici un hommage au sérieux à toute épreuve du grand-père Fontaine, et à la bonté et probité du petit-fils qui permet ainsi la publication souhaitée par Buonarroti de sa défense en entier.

\section{Un procès politique retentissant}

Pour comprendre la démarche de Buonarroti, il faut tout d'abord rappeler ici les circonstances très particulières du procès des babouvistes. Le 21 floréal an IV (10 mai 1796), les principaux animateurs du groupe des Égaux étaient interpellés par la police, suite à la dénonciation de l'agent

(1) Débats du procès instruit par la Haute-Cour de Justice, séante à Vendôme contre Drouet, Babeuf et autres, Paris, Baudoin, An V (1797).

(2) Philippe BuOnARROTI, Mémoires de F.-N. Gracchus Babeuf, précédés de l'ouvrage "Conspirations pour l'égalité, dite de Babeuf », Prospectus, Paris, Imprimerie de Carpentier-Méricourt, 1830. 
militaire Grisel : Didier, Germain, Darthé et Drouet appréhendés chez un militant, Babeuf, Buonarroti et Pillé, le secrétaire de la conjuration, surpris chez Babeuf ${ }^{3}$. Dans les jours et les semaines qui suivirent, le nombre d'incarcérations ne cessa d'augmenter, dans un contexte où le gouvernement faisait imprimer des affiches présentant les inculpés comme des anarchistes, des pillards, des assassins ${ }^{4}$, et où la presse directoriale se déchaînait contre ceux qu'elle accusait pêle-mêle d'être des agents de l'étranger, des terroristes ou même des royalistes ${ }^{5}$. Le gouvernement entendait en fait profiter pleinement de l'émoi suscité par la découverte d'une conspiration pour menacer l'ensemble de ses adversaires politiques démocrates. Des enquêtes s'ouvrirent un peu partout en France alors que parmi les individus arrêtés, un certain nombre n'avait aucune implication dans les projets des Égaux.

La présence parmi les inculpés du député Drouet avait mis les cinq Directeurs dans l'obligation constitutionnelle de réunir la Haute-Cour de Justice. Cette nécessité coupait cours à la possibilité de traduire les Égaux devant une commission militaire expéditive et contraignait les autorités à attendre la proclamation de cette instance judiciaire exceptionnelle pour juger en bloc tous les accusés. Cependant, elle offrait plusieurs avantages aux yeux du gouvernement. Tout d'abord, l'établissement d'un tribunal qui présentât toutes les apparences du respect de la légalité constitutionnelle, malgré les nombreuses irrégularités qui entâchèrent le processus réel de sa création, permettait de montrer à tous que le temps des tribunaux révolutionnaires et de la Terreur jacobine était passé, et de rassembler autour des Directeurs les possédants et les partisans de l'ordre en vue des élections de l'an V. Par ailleurs, elle permettait d'éviter que les accusés ne se pourvoient en cassation, dans la mesure où les jugements de la HauteCour devaient être exécutés dans les vingt-quatre heures, au mépris, par parenthèse, de la constitution elle-même. Last but not least, la Haute-Cour devant obligatoirement siéger à une distance éloignée de l'Assemblée nationale, les débats seraient hors de portée de la foule parisienne au sein de laquelle un certain nombre de dirigeants babouvistes était très populaire. Mais Paris devait être informé du déroulement des débats, le procès étant,

(3) Claude MAZAuric, « Carnot et les babouvistes » dans Jean- Paul CHARnAY (dir.), Lazare Carnot ou le savant citoyen, Paris, Presses de l'Université Paris-Sorbonne, 1990, p. 99-117.

(4) Laura MASON, «Le procès de Vendôme mis en imprimé », dans Babeuf et les babouvistes en leur temps, colloque des 16-17 octobre 1997, Saint-Quentin, Association Les amis de Gracchus Babeuf, 2000, p. 117.

(5) Jean-Marc SchIAPPA, Les babouvistes, Saint-Quentin, Association Les amis de Gracchus Babeuf, 2003, p. 368-369. 
pour la première fois dans l'histoire judiciaire française, transcrit par des copistes-sténographes et publié par l'imprimerie nationale, ce qui allait donner à l'événement, que nombre de journaux commenteraient, un écho très large. De toute évidence, ce tribunal d'exception et son verdict devaient aux yeux de son principal maître d'oeuvre, le Directoire et notamment Carnot, revêtir un caractère exemplaire et marquer les esprits à droite comme à gauche.

Les accusés furent laissés au secret jusqu'à leur transfert à Vendôme, dans la nuit du 9 au 10 fructidor an IV (27 août 1796). Dès les premiers jours suivant son arrestation, Babeuf revendiqua hautement la conspiration et en soutint la légitimité. Alors que Drouet était parvenu à s'évader, peutêtre avec l'aide des autorités elles-mêmes, les autres accusés gagnèrent Vendôme «dans des cages grillées construites exprès pour les donner, comme des bêtes féroces, en spectacles aux ennemis de l'égalité », et suivis à pied par les femmes, filles et soeurs des accusés qui elles aussi « essuyèrent fréquemment les rigueurs de l'atmosphère et les sarcasmes des aristocrates $»^{6}$.

Ce n'est qu'une fois transférés que les Égaux purent mettre au point un système commun de défense, essayer de récuser une partie des jurés proposés comme la loi le leur permettait. Les interrogatoires, l'élection des juges et des jurés durèrent jusqu'en octobre 1796 ; le procès ne s'ouvrit finalement qu'en février 1797.

C'est donc le 2 ventôse an V que « commença l'un des procès les plus extraordinaires de l'histoire $\gg^{7}$ par son ampleur et sa portée. Les accusés étaient au nombre de soixante-quatre, dont dix-huit contumaces, même si d'après Buonarroti dans sa relation postérieure de l'événement, seuls vingt-cinq d'entre eux avaient effectivement pris part aux préparatifs de la Conjuration, et cinq les y avaient aidés indirectement, les autres y étant complètement étrangers. Le procès public fut extrêmement long, avec plus de cinquante séances, du 2 ventôse au 7 prairial (25 mai 1797). Les débats se déroulèrent le plus souvent dans un climat houleux, avec des juges et des accusateurs nationaux (Viellart et Bailly) ouvertement hostiles aux accusés, une foule nombreuse dans cette salle d'audience gardée militairement, et des chefs de file comme Babeuf, Germain, ou d'autres, qui multiplièrent les incidents de séance, interrompant les débats, invectivant, protestant, chantant des chants républicains souvent sous les applaudissements d'un

(6) Philippe Buonarroti, op. cit. p. 15. 1962, p. 214

(7) Claude MAZAURIC, Babeuf et la Conspiration pour l'Égalité, Paris, Éditions Sociales, 
public favorable. Par l'intermédiaire d'un républicain fervent, ami de Babeuf et de sa famille, Pierre-Nicolas Hésine, et de son Echo des hommes vrais et sensibles, journal de la Haute-Cour de Justice, dont soixantetreize numéros parurent tout au long du procès, les accusés disposaient non seulement d'un journal qui leur était favorable mais encore, selon des recherches érudites synthétisées par Claude Mazauric, « d'une tribune imprimée pendant toute la période de leur incarcération dans la ville de la Haute-Cour $»^{8}$, ils faisaient probablement passer hors de la prison des écrits par leurs parents ou leurs défenseurs, qui les transmettaient au journaliste et à sa femme.

Le procès connut un certain nombre de temps forts. D'abord la Cour décida de faire comparaitre Grisel, ce qui était illégal puisqu'il était le dénonciateur. Sa déposition hargneuse dura deux jours et suscita de véhémentes protestations de Babeuf et Germain. Dans un tout autre registre, l'attitude de Meunier et Barbier, deux membres de la Légion de police qui vinrent à la barre rétracter des aveux qui leur avaient été extorqués sur la Conjuration, et écopèrent plus tard pour cela de vingt ans de fers, suscita une forte émotion. Enfin, le 7 prairial, après des délibérations pendant lesquelles s'étaient exercées les plus fortes pressions sur les rares jurés susceptibles de prononcer l'acquittement général, notamment sur le plus républicain d'entre eux, Gaultier de Biauzat, ancien Constituant représentant du Puy de Dôme ${ }^{9}$, le jury de Vendôme acquittait presque tous les accusés, mais condamnait sept d'entre eux (Buonarroti, Germain, Moroy, Cazin, Blondeau, Mennessier, Bouin) à la déportation, et deux d'entre eux, Babeuf et Darthé, à la peine capitale. Un tumulte s'ensuivit, pendant lequel Babeuf et Darthé se plongèrent un stylet dans la poitrine. Leur tentative de suicide réussit-elle ? Les témoignages divergent sur la question. Le lendemain même, morts ou moribonds, ils furent conduits à l'échafaud et exécutés.

\section{La stratégie de défense des accusés de Vendôme}

Une importante particularité du déroulement de ce procès politique tient au choix que les accusés firent de leur système de défense, qui consista globalement à nier la réalité de la Conjuration dont on les chargeait, et à réduire leur activité commune à celle d'une "Société des démocrates », un club de réflexion et de propagande républicaine sans volonté réelle

(8) Claude MAZAURIC, op. cit. p. 212.

(9) Philippe BouRdin, entre autres travaux, Des lieux, des mots, les révolutionnaires.., Clermont-Ferrand, Université Blaise Pascal, 1995, p. 410. 
de passage à l'acte. Ainsi, malgré la dénonciation de Grisel, malgré le grand nombre de documents manuscrits saisis au domicile de Babeuf et constituant autant de preuves accablantes, les plaidoiries s'empêtrèrent souvent dans des justifications maladroites, des interprétations sophistiques et peu convaincantes des pièces les plus compromettantes; mis à part celle de Babeuf lui-même, elles atténuèrent globalement la portée politique du projet des Égaux en mettant notamment sous le boisseau le thème incendiaire de « la communauté des biens et des travaux » qui en était le fondement doctrinal.

Une telle stratégie, produit d'une délibération collective menée dans la prison de Vendôme, est liée aux grands contrastes dans l'implication concrète et morale des différents accusés. Elle reflète très certainement certaines divisions politiques et idéologiques entre ceux pour qui «le communisme était le moteur de l'action » et ceux pour qui il «n'était peut-être pas un objectif qui valût la peine de s'exposer à la peine maximum ${ }^{10}$. Les échos dans la prison de la tentative avortée du «coup de Grenelle », (23-24 fructidor, 9-10 septembre 1796), lors de laquelle des partisans babouvistes, avec d'autres démocrates attachés au souvenir de l'an II, avaient essayé de soulever la garnison de Grenelle, et s'étaient fait brutalement réprimer, dut renforcer la volonté d'édulcorer le sens de l'activité du groupe. Le cercle restreint des Conjurés les plus convaincus, en se ralliant à ce choix qui pourtant minimisait la portée exemplaire de leur geste et blessait leur foi, chercha sans doute à protéger la majeure partie de leurs camarades ; ce faisant, les conjurés reportaient toute la rigueur vengeresse des juges sur leur propre personne dont les actes insurrecteurs et autres écrits de leur main montraient sans rémission possible la réalité du projet politique et social. Deux d'entre les prévenus, Darthé et l'ancienne adhérente de la société des républicaines révolutionnaires, Sophie Lapierre, refusèrent de se rallier à la décision commune : tous deux rejetèrent la compétence de la Haute-Cour puis s'enfermèrent dans un silence méprisant pendant toute la durée du procès.

On a beaucoup discuté de la pertinence de cette orientation globale. Incontestablement, elle a embarrassé les babouvistes les plus convaincus, les empêchant de défendre leurs positions avec toute la clarté et la cohérence souhaitées, en appelant au rassemblement clairvoyant de tous les démocrates, les contraignant simultanément à adopter une posture de rêveurs impuissants ou d'agitateurs dangereux. « Aucun accusé n'était plus 
que Babeuf gêné dans sa défense $»^{11}$ dit en particulier Buonarroti, Babeuf que tout accusait et que son naturel bouillant et héroïque avait poussé dans les premiers moments à assumer fièrement ses actes. Georges Lefebvre notamment critiqua ce choix, considérant que l'attitude de Darthé avait été « la plus conséquente et la plus digne » et que tout « cela ne servit du reste à rien $»^{12}$, puisque les condamnations tombèrent malgré tout.

Cette interprétation cependant ne prend pas en compte, comme Claude Mazauric le souligne, le fait que la majeure partie des accusés fut effectivement acquittée en fin de compte. Ian Birchall va plus loin, affirmant que Buonarroti lui-même « a exagéré la faiblesse de la stratégie : elle n'a pas empêché les accusés d'utiliser la Cour comme tribune pour un puissant exposé de leur politique $»^{13}$. Rappelons enfin, que pour pouvoir prononcer les condamnations, les juges de la Haute-Cour furent réduits à employer une méthode illégale, ajoutant rétrospectivement à l'accusation première de conspiration contre le gouvernement, celle d'avoir cherché, par des discours ou des actes, à rétablir la Constitution (abolie) de 1793. Concernant la première charge, les accusés avaient obtenu gain de cause, puisque les jurés avaient conclu à un non-lieu. Malgré le sacrifice de Babeuf, chef incontesté de la Conjuration et de Darthé, qui du fait de son attitude au procès, comme de sa collaboration passée avec Joseph Le Bon au tribunal révolutionnaire d'Arras pendant la Terreur, était une victime toute désignée, le «pieux mensonge » des accusés de Vendôme était donc légitimé a posteriori par son résultat. La plupart des accusés avaient été épargnés.

\section{Le cas Buonarroti}

Contrairement à celle de ses deux infortunés camarades, Babeuf et Darthé, la présence de Buonarroti sur le banc des accusés était problématique pour la Haute Cour. À cette époque, l'homme, issu d'une illustre famille toscane, était en effet (déjà) une grande figure politique. De Corse, il s'était embarqué avec Bonaparte pour l'expédition de Sardaigne en janvier 1793. Par la suite, il avait été chargé par la Convention d'une mission de réorganisation administrative de la Corse, qu'il n'avait pu mener à bien, puis avait été nommé commissaire national à Oneilla en Ligurie, au-delà

(11) Philippe BuONARROTI, op. cit., p. 30.

(12) Georges Lefebvre, La France sous le Directoire, Paris, Éditions Sociales, 1977, p. 204-205.

(13) Ian BIRCHALL, "The Vendôme defence strategy », Journal for Eighteen Century Studies, vol. 20, September 1997, p. 145. Traduction personnelle. 
de la frontière italienne, au printemps 1794. Au moment où la Conjuration avait été découverte, il venait d'être promu commandant pour être partie prenante de l'expédition militaire en Italie confiée à Bonaparte. Certains bonapartistes dans l'armée, pas tous et peut-être pas Bonaparte lui-même, cherchaient certes à l'éloigner, mais pas à l'éliminer.

Au procès, Philippe Buonarroti montra plus de retenue que les autres dirigeants : il interrompit rarement les débats, ne participa pas aux tumultes. Il intervint toujours en montrant une connaissance approfondie des lois, tentant par exemple sans succès d'empêcher l'audition de Grisel le 7 ventôse. Lorsque son cas fut abordé le 14 germinal, il prononça une profession de foi personnelle, dans laquelle il rappelait les services rendus par lui à la cause de la Révolution Française, son attachement précoce aux principes rousseauistes et républicains et évoquait une première fois comment « la constitution de 1793 devint [sa] religion $»^{14}$. Le révolutionnaire cherchait à rappeler au jury qu'il avait en tous points mérité de la patrie, ce qui constituait une défense justifiée et n'impliquant pas de compromission par ailleurs.

Son grand discours de défense, qui constitue les deux premières parties de notre manuscrit, prononcé le 21 floréal, ne fera pas non plus état de convictions clairement hostiles au droit de propriété. On y trouve un Buonarroti tentant, après Babeuf et avec les mêmes explications contournées, d'atténuer la signification insurrectionnelle des pièces à conviction, et d'accréditer l'idée d'un groupe de promotion d'idées démocratiques et humanitaires sans portée pratique immédiate et sans moyens réels. Il insiste beaucoup sur la sensibilité de ces patriotes aux malheurs publics et à la misère du peuple. Mais en même temps, Buonarroti revenant sur les circonstances des années de révolution, et notamment sur la période de la Convention montagnarde puis thermidorienne, se livre à une défense des mesures de Terreur de l'an II, rappelant les difficultés exceptionnelles rencontrées par la jeune République ; il réaffirme le caractère démocratique de la Constitution de 1793 (An premier de la République) et l'importance du principe de souveraineté du peuple que ses dispositions consacrent.

La partie inédite, dont nous reproduisons ici la partie la moins technique et la plus dense d'un point de vue politique ${ }^{15}$, intitulée « Fond de la Conspiration », reste dans le même esprit. Malgré les précautions de langage qui l'entourent, on peut la lire comme l'affirmation maintenue d'un

(14) Débats du Procès..., op. cit., t. III, p. 217.

(15) Le texte sera disponible dans sa totalité sur le site de la revue. 
certain nombre d'idées qui ont incontestablement fondé la Conjuration. Tout d'abord, on y retrouve la volonté de rétablir en les prolongeant les mesures économiques et sociales prises sous la Terreur en faveur des catégories sociales les plus démunies, même si Buonarroti avait retranché de ces propositions leur caractère transitoire vers la communauté des biens : réquisition des boulangeries et des armureries confiées à la garde du peuple, distribution des biens nationaux aux nécessiteux et aux défenseurs de la patrie ; adoption par la nation des veuves et des orphelins des soldats républicains tombés au combat, etc. Encore une fois, l'orateur revint sur les circonstances respectives des votes des Constitutions de 1793 et de 1795, afin de montrer l'incontestable supériorité, en matière de légitimité démocratique, de la ratification bien plus massive de la première. En termes certes choisis, il suggérait néanmoins de manière claire que son abandon au profit de la constitution de l'an III était un déni de souveraineté populaire, et que de véritables patriotes ne pouvaient que le contester. Il cherchait à démontrer que de telles entorses aux règles républicaines ne pouvaient qu'encourager les menées royalistes. Plus que jamais, il s'agissait de prendre au mot les réprésentants de l'autorité républicaine et de faire des jurés les arbitres des contradictions du Directoire par rapport aux principes qu'il affichait. Buonarroti l'écrivit plus tard : il cherchait à l'époque à s'adresser « à la vertu des jurés » et à leur « patriotisme ». De ce point de vue, on le voit, il ne trahissait pas ses engagements passés, et malgré les euphémismes et les concessions indéniables, il continuait d'assumer une part du fond politique babouviste.

Comment expliquer que cette dernière partie du discours n'ait finalement pas été prononcée ? En tout état de cause, la loi ne prévoyait pas que les prévenus puissent formuler une réplique à la réponse de l'accusateur national. Mais on a suffisamment évoqué les multiples libertés prises à l'égard de la procédure, aussi bien par la Cour que par les accusés euxmêmes dans une certaine mesure, et le caractère exceptionnel du procès. Une autre explication peut peut-être être avancée ${ }^{16}$. En effet, les accusés avaient choisi d'assurer eux-mêmes leur défense, mais on leur avait adjoint un défenseur en la personne de l'avocat Réal, lui-même proche de Barras, l'un des cinq Directeurs, mais aussi de Bonaparte dont il sera, l'Empire proclamé, l'un des très hauts serviteurs. Les cinq ont pu chercher à sauver la tête de Buonarroti par son intermédiaire. Il est ainsi permis de se demander 
si l'avocat, prévenu que les jurés auraient finalement à se prononcer sur le délit de tentative de rétablissement de cette constitution de 1793 qu'ils avaient en horreur, n'a pas dissuadé Buonarroti de réitérer en des termes sans équivoque son attachement à cette dernière, aggravant par là son cas. Quoi qu'il en soit, les Jurés trouvèrent finalement des circonstances atténuantes à celui dont la main avait sans doute rédigé, après celle de Babeuf, le plus grand nombre des documents jugés « compromettants ». Nous concluerons donc sur cette interrogation en suspens, laissant au lecteur la liberté de statuer par lui-même sur ces questions à la lecture de ce texte inconnu et sauvé de l'oubli.

\section{Fond de la Conspiration}

[Cette partie du manuscrit constitue le coeur de la défense de Buonarroti et se trouve en dernière partie de la « Réplique à la réponse de l'Accusateur national ». Elle comporte 25086 signes. Les biffures et les mots en gras sont de la main de Buonarroti. Nous indiquons entre crochets les césures de pages. Les mots soulignés dans le manuscrit sont en italiques ici. Les passages ajoutés sont précédés de la mention «passage ajouté » et mis en italiques.]

[p. 18] S'il était possible de voir dans cette affaire une véritable conspiration les preuves ne pourraient sortir que de l'arrangement des pièces dans l'ordre imaginé par les accusateurs nationaux ; or on a vu dans le débat de Babeuf combien il est naturel de donner à beaucoup d'entre elles un sens et un objet différents de celui qu'ils leur prêtent, et alors on sent aisément combien il est facile de faire crouler cette monstrueuse statue à pieds d'argile.

C'était à celui qui connait la source et le véritable objet de ces pièces à vous en donner l'explication : vous avez remarqué, Citoyens Jurés, que sa défense a surtout établi la vérité de quatre points principaux : savoir la pureté des [p. 19] intentions de la société des Démocrates dont il vous a parlé et leur extrême sensibilité pour tous les maux qui avaient fondu sur la patrie : une frayeur constante des manoeuvres sans cesse ourdies par le royalisme, dont la plus à redouter était l'adresse de profiter de l'esprit du gouvernement actuel pour ramener insensiblement l'État à la Monarchie ; la tâche qu'elle s'était imposée de recueillir toutes les idées récentes et 
éloignées relatives à l'essence et à l'établissement de la Démocratie : l'interruption et la divergence de leurs $\mathbf{s e s}^{17}$ opérations.

Quoi qu'en dise l'accusateur national, je crois que le compte que j'ai rendu des pièces de mon écriture aura arrêté l'attention des Jurés. Ils auront vu surtout combien sont frêles les inductions qu'il a prétendu tirer de celles que j'ai écrites à la maison d'arrêt du Plessis ${ }^{18}$ : dès qu'une partie de l'édifice croule tout le reste ne peut tarder [p. 20] à tomber en ruine.

Constant dans l'ordre de discussion que j'ai suivi dans mon discours du 21 Floréal, j'adopte un instant pour vrai l'arrangement des accusateurs : et je passe à peser les objections qu'ils ont opposées à la justification du résultat de leur propre système.

Ces mots pillage et massacre vous sont bien chers ; c'est à l'aide de vos phrases hypocrites, de votre feinte indignation que vous espérez exalter l'opinion et exciter contre nous le ressentiment universel ; vous espérez surtout, et ceci est d'une grande leçon pour le Peuple, vous espérez surtout faire détester la Révolution, et ramener tous les esprits à préférer une tranquille Servitude à la méfiante Liberté. Nouveaux Appius ${ }^{19}$ vous ne voyez dans les malheurs de la multitude que des reproches perpétuels et vous craignez sans cesse en elle les ressentiments que vous avez trop mérité.

[p. 21] Vous dîtes que le pillage était moyen et but de l'insurrection qu'on projetait : mais accordez de grâce cette constante et ridicule prétention avec l'article formel de l'acte insurrecteur (acte qui, s'il y a eu conspiration doit être regardé comme le dépôt final des intentions des conspirateurs) qui met expressément les propriétés publiques et particulières sous la sauvegarde du peuple; vous ne parlez jamais de cet article qui ne cadre pas avec vos vues meurtrières. Vous n'êtes donc pas d'accord avec vos consciences quand vous prétendez que la possession qu'on insinuait au Peuple de prendre des magasins publics ou privés contenant des vivres ou des munitions de guerre était une provocation au pillage, tandis qu'il est constant et par l'article susdit et par la vertu connue du Peuple de Paris

(17) Les biffures se trouvent dans le manuscrit; les mots en gras sont de la main de Buonarroti lui-même.

(18) Buonarroti avait été arrêté le 5 mars 1795 suite à la plainte d'un noble sarde qui l'accusait d'avoir fait saisir ses biens dans l'exercice de ses fonctions à Oneilla. C'est en prison au Plessis qu'il fit la connaissance de Babeuf et qu'il fut probablement gagné aux idées communautaires de ce dernier.

(19) Il s'agit peut-être de Claudius Appius Regillanus, decemvir préposé à la rédaction des lois sous l'empereur Tibère, qui provoqua une rupture entre le Sénat et le Peuple à cause de ses mauvaises moeurs. 
expérimenté en plusieurs autres circonstances que les propriétés confiées à sa garde ne pouvaient pas avoir de plus sûr garant.

[p. 22] Faut-il aussi que je définisse le pillage ? Faut-il aussi que vous vous serviez pour nous accabler de chacun des mots que vous ne voulez pas définir ? Le pillage n'est-il pas dans la dispersion des biens meubles sans aucune indemnité pour le propriétaire ? Et pouvez-vous en voir un dans les réquisitions aux boulangeries de cuire du pain et aux armureries de donner leurs armes sous la condition expresse de les payer sur leur seule déclaration? Art. 15 de l'acte insurrecteur ${ }^{20}$.

Y a-t-il la moindre idée de pillage dans les bienfaits qu'on se proposait de répandre aux frais de la République sur les infortunés, soit pour la distribution à eux et aux défenseurs de la Patrie des biens nationaux, soit en les logeant dans les maisons nationales, soit en les habillant aux dépens de la nation, soit en rendant gratuitement au Peuple les effets déposés au Mont-de-Piété, [p. 23] soit en adoptant les épouses et les enfants des citoyens péris pour la défense de la Liberté ?

$\mathrm{Ah}$ ! La conception seule de tels actes est faite pour honorer le coeur de ceux qui les ont écrits : et vous avez beau blasphémer, les hommes impartiaux sauront apprécier la rage avec laquelle vous vous acharnez à les déshonorer.

Toutes les dispositions de cette nature étaient subordonnées à la volonté du Peuple ; ceux qui les traçaient sur le papier auraient eu tout au plus le mérite de les lui avoir proposées ; elles portaient toutes sur les bien nationaux, qui, oh ! Honte de la Révolution, ont fini par passer à vil prix dans les mains des voleurs publics, des spéculateurs sur la misère des français !... Ah ! Certes, le luxe de quelques fripons vaut mieux pour l'ordre que quelques légers secours distribués sagement à une multitude d'infortunés que la Révolution [p. 24] et la méchanceté avaient dépouillés de toute ressource. Je le répète hautement le mot insensé, affreux de pillage n'a été que dans les Lettres et dans le coeur de l'épouvantable Grisel ${ }^{21}$.

Et tant d'éclatants témoignages de sensibilité, de compassion serontils perdus pour la conscience des Jurés ? La compassion est pour quelques âmes une passion forte à laquelle les méchants ne croient pas ; mais vous, Citoyens, vous saurez démêler ce sentiment qu'on veut si étrangement

(20) Reproduit dans Philippe BUONARROTI, op. cit. p.169 : « Tous les boulangers seront en réquisition pour faire continuellement du pain, qui sera distribué gratis au peuple; ils seront payés sur leur déclaration ».

(21) En effet, le terme se trouve dans une « lettre » de Grisel destinée à la propagande parmi les soldats, et reproduite dans Philippe BUONARROTI, op. cit. p. 182-185, attestant le rôle d'agent provocateur joué par le dénonciateur. 
défigurer, vous sentirez, je l'espère, par votre propre expérience qu'il peut devenir impérieux et déterminant pour les hommes qui ont la conviction intime de la dignité du Peuple et qui se sont accoutumés à réfléchir sur eux-mêmes les innombrables douleurs qui l'accablent.

Toutes les fois qu'on vous parle raison vous criez massacre, sang, terreur, en vérité si je ne [p. 25] me connaissais pas vous m'auriez convaincu que je suis un cannibale : il y a une singulière remarque à faire, c'est que chez toutes les nations toutes les fois que l'on a réclamé les droits de la Nature ceux qui ne s'en accommodaient pas, et qui se sentaient en faute ont toujours essayé d'effrayer la masse en criant à l'assassinat et à la fureur : semblables à des conquérants jaloux ils ne voyent que révolte dans les moindres plaintes de ceux qu'ils oppriment.

À entendre les accusateurs on ne sait pas où se serait arrêté le carnage : Députés, Directeurs, fonctionnaires, Propriétaires tous devaient être immolés. Réduisons cela à sa juste valeur, et abordons à regret cette dégoûtante discussion.

Une pièce de la procédure a donné aux accusateurs nationaux lieu d'effrayer l'imagination des Jurés et [p. 26] du Peuple : je veux parler de la fameuse pièce dite tuer les $\operatorname{cinq} q^{22}$. On a prétendu qu'elle était le résultat des délibérations d'une assemblée de conjurés, et comme elle contient des mesures violentes on en a conclu que telles étaient les vues du prétendu Directoire insurrecteur.

J'observe qu'il est souverainement injuste d'attribuer à ce Directoire dans le cas qu'il y en ait eu un les idées qu'il n'a pas adoptées, et qui ne peuvent se retrouver que dans les actes qui paraissent évidemment lui appartenir tels que l'acte insurrecteur où il faut chercher seulement le dernier résultat de ses projets.

Cette pièce tuer les cinq est évidemment un avis individuel fruit d'une imagination exaltée indignée contre les injustices dont tout le monde était spectateur : ce que j'avance est démontré [p. 27] par cette phrase de la p. 422 vol. $1^{\text {er }}:$ Je pense qu'il est politiquement essentiel. Cette manière de s'exprimer annonce bien clairement que la pièce entière ne contient que les extravagantes rêveries d'un seul homme ; je reviendrai d'ailleurs sans cesse sur ces traits qui ont effacé le mot qu'on veut avoir été tuer, traits qui me paraissent le témoignage le plus sensible du dégoût que le contenu de

(22) Ce fameux texte constitue les $34 \mathrm{e}$ et $35 \mathrm{e}$ pièces de la $8^{\mathrm{e}}$ liasse, dans Copie des pièces saisies dans le local que Babeuf occupait lors de son arrestation, Paris, Imprimerie nationale, an V, $\mathrm{t}$. I, p. 238-242. Il est de la main de Darthé et a constitué un point d'appui majeur de l'accusation contre les babouvistes, parce qu'il contenait cet appel au meurtre. 
cet écrit avait inspiré à ses lecteurs, ou peut-être à l'auteur lui-même. Il faut donc l'écarter de toute discussion.

Les Députés, dîtes-vous, et les Directeurs devaient être jugés par le Peuple d'après l'acte insurrecteur et vous concluez de là qu'ils étaient voués à la mort ; mais par quel magique événement transformez-vous l'idée de jugement en celle de mort ? N'est-ce pas au Peuple à juger ses mandataires ? N'est-ce pas le Peuple qui nous juge ici par ses Représentants, n'était-ce pas [p. 28] le Peuple qui jugeait à Rome les citoyens ? Et de quel droit transformez-vous en Bourreaux les citoyens français ? Quand les auteurs des pièces ont voulu la mort ils ont écrit sans mystère la mort, tuer; dès qu'ils se sont servis du mot juger il faut conclure, la Justice vous le commande, qu'ils faisaient une grande différence entre le jugement et la mort.

La mort dans l'acte insurrecteur et même dans la 9e pièce de la 2e liasse $\mathrm{e}^{23}$ est une simple menace faite à ceux qui contre la volonté du peuple auraient exercé des fonctions, ou se seraient opposés à ses vues : quelle énorme différence ! Au lieu de voir dans ces dispositions le but unique, affreux, coupable d'exterminer pour assouvir la vengeance, on ne retrouve que des menaces pour écarter l'opposition à des opérations pour lesquelles la volonté [p. 29] du Peuple était la condition suprême : cessez donc, cessez vils calomniateurs de la Révolution, cessez âmes de glace trempées au crime, des peindre les plus chauds amis de l'humanité sous les couleurs odieuses qui conviennent seulement à vos froids calculs.

À vos déclamations, à vos furieux efforts contre la Philosophie qui irrite votre orgueil, j'opposerai sans cesse ces mots mémorables écrits de la main de Babeuf, de celui que vous vous obstinez à peindre comme le chef de la Conjuration, non dans une pièce destinée à devenir publique mais dans l'épanchement secret de son âme : rendre les chefs responsables $d u$ sang qui sera répandu ${ }^{24}$, p. 68 du 1er volume.

Où avez-vous lu que les Listes qui sont au procès fussent des listes de proscription? Ne pouvait-on pas avoir mille motifs raisonnables [p. 30] ordinaires de connaitre les bons, ou les mauvais Républicains?

(23) Copie des pièces..., op. cit., t. I, p. 26 : «Tuer sur-le-champ tout député, directeur, administrateur, juge, officier ou fonctionnaire public, qui paraitrait pour donner des ordres, ou pour l'exercice d'une fonction. Arrêter tout député ou directeur trouvé dans les rues, le faire conduire à son poste pour y être jugé ».

(24) C'est effectivement ce que l'on trouve dans la $23^{\mathrm{e}}$ pièce de la $5^{\mathrm{e}}$ liasse, de la main de Babeuf. Tout au long du processus révolutionnaire, le tribun a manifesté son horreur du sang versé, dans des écrits publics comme dans sa correspondance personnelle. 
De quel droit vous permettez-vous d'effrayer imprudemment les Propriétaires dont les biens étaient expressément mis sous la sauvegarde du Peuple par l'acte insurrecteur?

Et que serait-ce au fond les menaces et même les projets d'une sévérité outrée si la conspiration dont on en fait le moyen et le but, est hors de l'animadversion de la Justice par cela seule que dénuée de la possibilité de réalisation, elle n'exposait la société à aucun danger, et n'était pas ainsi une véritable conspiration.

J'avais démontré, je pense à la séance du 21 que ceux dont on veut faire des conjurés n'avaient ni les soldats, ni les armes, ni l'argent, ni la puissance [p. 31] moyens indispensables pour l'exécution de leurs projets.

L'accusateur voit des moyens efficaces dans les notes des magasins contenant des armes et des provisions : ces notes indépendamment des explications qui leur ont été données prouvent tout au plus que les prétendus conjurés n'avaient pas un fusil, pas une pique à leur disposition.

Il voit des moyens alarmants dans les nomenclatures d'hommes propres à commander ; rappelez-vous, citoyens Jurés, qu'on a donné à ces listes des explications très vraisemblables auxquelles vous ne pourriez vous dispenser d'avoir égard sans une extrême injustice : on a dit qu'elles avaient été rédigées en Vendémiaire ${ }^{25}$ pour fournir à la Convention des défenseurs ; les événements de cette époque ne sont-ils pas assez connus, les dangers que courait alors la République ne sont-ils [p. 32] pas écrits en caractères de sang dans les pages de notre histoire ? Mais fussent-elles ces listes telles que vous le prétendez où est le fil secret par lequel on devait faire mouvoir les hommes qui y sont inscrits, où sont les engagements, les promesses, le secret, les assemblées, le concert sans lesquels il est impossible de pouvoir compter sur la volonté d'autrui ?

L'exaspération du Peuple, dîtes-vous est le grand moyen par lequel les conjurés espéraient accomplir leur funeste dessein : la famine et les malheurs causés par le maximum, les réquisitions et la terreur étaient les plus puissants ressorts dont les conspirateurs voulaient profiter.

Il faut rectifier sans cesse une erreur funeste par laquelle on a depuis longtemps bouleversé toutes les idées, perverti les [p. 33] esprits, fait oublier l'histoire et contre-révolutionné la France. Ce n'est pas au maximum et aux réquisitions qu'il faut attribuer la famine et les malheurs publics, c'est à la conspiration puissante de l'intérieur qui arma contre le Peuple français

(25) Le 13 vendémiaire an IV, une insurrection royaliste dans Paris menaça la Convention qui dut réarmer des patriotes poursuivis ou incarcérés après Thermidor. Il y eut environ 2 à 300 tués et blessés de chaque côté, et la répression qui s'ensuivit fut modérée. 
tous les tyrans ligués de l'Europe qu'il faut imputer les efforts qu'on fut obligé de faire pour les repousser; et si de mauvais citoyens voyaient avec indifférence les dangers de la Liberté, fallait-il la laisser périr, fallait-il arracher au malheureux qui versait son sang jusqu'à son dernier haillon ? On demanda à celui qui en avait le superflu qu'il devait à la Patrie, et celui-ci avait souvent la barbarie de le refuser ! Fallait-il comme sous le régime de la terreur réactionnaire discréditer, par une énorme émission, la monnaie nationale et faire peser uniquement sur la classe laborieuse tous les frais de l'état ? Car elle seule a payé le discrédit de la fortune publique : avant [p. 34] on avait demandé aux riches leur superflu, après on arracha aux pauvres leur nécessaire : la vérité est que l'une et l'autre mesure dont on apprécie l'énorme différence furent commandées non par un système fantastique de brigandage mais par les manoeuvres dangereuses et toujours renaissantes des ennemis extérieurs et intérieurs de la République.

L'exaspération du peuple était juste : les écrivains qui faisaient retentir la France de ses malheurs étaient ses répétiteurs : l'injustice de l'autorité fut toujours de s'en prendre à ceux qui publient la vérité que chacun nourrit secrètement dans son coeur.

Mais Et cette exaspération, un peu de sensibilité l'aurait éteinte, un rien l'étouffait : les groupes de Germinal ${ }^{26}$ s'évanouissaient à la vue d'une patrouille, la Société du Panthéon ${ }^{27}$ disparut à la lecture d'un arrêté : il ne restait aux [p. 35] hommes sensibles que des regrets superflus, des plaintes inutiles.

Ouvrez, je le répète, ouvrez la correspondance que vous attribuez aux prétendus agents, prenez même le prétendu rapport des agents militaires ; vous y lirez que le Peuple ne pouvait se mouvoir sans les troupes, que celles-ci ne pouvaient être déterminées que par l'argent... et l'argent, les trésors de la conspiration étaient... six mille livres en Assignats, deux pamphlets, des voix perdues, une austérité ridicule, un zèle inutile.

Comparez, si vous voulez la situation des Patriotes en Prairial de l'An III ${ }^{28}$ à celle de Floréal de l'An IV ; alors le Peuple avait encore ses

(26) Journées des 12 et 13 germinal an III : manifestations des sans-culottes parisiens pour obtenir du pain et la Constitution de 1793. Sa répression consolida le pouvoir des thermidoriens et priva de dirigeants le mouvement populaire, notamment à Paris.

(27) Lieu où se rassemblèrent (et s'affrontèrent) les patriotes « de gauche » à partir du 25 brumaire an IV. C'est Bonaparte qui finit par obtenir du Directoire l'ordre de sa fermeture le 9 ventôse.

(28) Le 1er prairial avait été une journée d'insurrection sans-culotte avec les mêmes mots d'ordre qu'en germinal, dans un contexte où la disette sévissait. La foule envahit la Convention mais en fut repoussée dans la nuit. Les députés montagnards qui avaient fait voter des décrets favorables aux insurgés furent arrêtés. Dans les jours qui suivirent, la répression permit d'éliminer les derniers représentants de la Montagne et de défaire le mouvement populaire. 
assemblées, tous les citoyens étaient armés, les Sections de Paris gardaient leur artillerie, il avait grand nombre de partisans dans la Convention et dans les autorités ; en Floréal de l'An IV au contraire plus d'assemblées, plus d'armes, point d'argent, point de partisans [p. 36] dans l'autorité, l'inquisition la plus sévère, la terreur la plus révoltante sur tout ce qui avait l'air de la Démocratie.

Oh vous qui tout en convenant qu'il n'y a pas de conspiration criminelle là où il n'y a pas de danger pour le gouvernement, voulez qu'il y en ait une dans cette affaire, dîtes-nous comment il n'y eut pas de conspiration en Vendémiaire de l'An IV quand les conjurés avaient à leur disposition

40000 baïonnettes, quand le sang français coula dans les rues de Paris ; jusqu'à ce que vous nous ayez bien expliqué cet étonnant mystère nous crierons à l'injustice toutes les fois que vous voudrez voir conspiration dans la misérable affaire du 21 Floréal $^{29}$.

Nous voilà enfin au point le plus important de la discussion : il présente les armes les plus victorieuses contre les objections de l'accusateur.

Je me suis placé à la séance du 21 dans [p. 37] l'hypothèse que les auteurs des pièces de la procédure désiraient réellement ramener le Peuple à l'établissement de la Constitution de 1793, et j'ai conclu que même dans cette supposition et d'après les circonstances résultantes du procès ils ne seraient pas criminels.

J'ai besoin d'une grande latitude et de beaucoup d'attention pour développer et rendre sensible à cet égard la justification de l'affaire sous ce point de vue.

Fixez avant tout, je vous prie votre attention sur la distinction que j' ai faite à la séance du 21 entre la Constitution de 1793 et le Gouvernement de $1793^{30}$ : cette constitution n'a jamais été en activité, il me semble même que ceux qui la caractérisent si souvent d'anarchique ne la connaissent pas. Il faut donc se bien garder d'attribuer à cette constitution rien de tout ce qu'on peut croire avoir à reprocher au Gouvernement révolutionnaire.

Cette distinction bien posée, je dis que les [P. 38] intentions de ceux qui ont voulu la rétablir ne furent pas criminel [sic] s'ils ont cru fermement, décisivement, de bonne foi qu'elle était la véritable loi de la France, qu'elle consacrait un droit inaliénable de la Nation.

(29) Il s'agit du 21 floréal an IV, jour de l'arrestation des chefs babouvistes, à distinguer du 21 floréal an $\mathrm{V}$, jour de la première plaidoirie de Buonarroti.

(30) La Convention avait en effet ajourné jusqu'à la paix l'application de la Constitution qui venait d'être adoptée. 
C'est ici le cas d'observer qu'en matière de conspiration l'inspection du juge doit se porter et sur ce qu'on voulait renverser, et sur ce qu'on voulait substituer : si les vues personnelles sont démontrées le crime est certain : mais si la conviction du bien est constante... Si vous ne pouvez pas douter que le coeur de l'accusé ne palpitait que pour la justice, que son esprit en avait soigneusement mesuré les préceptes pourriez-vous le frapper sans effroi?

Ce qui constitue la criminalité en matière de conspiration est la science de la légitimité de l'autorité contre laquelle on dirige ses plans : si cette science n'existe pas, s'il y a une [p. 39] conviction contraire fondée sur les raisonnements les plus séduisants, sur les calculs que l'homme le plus prudent ne peut pas se dispenser de reconnaitre pour vrai [sic] avouez-le, il y aura peut-être erreur, mais pas criminalité... Vous pourrez argumenter, convertir, mais non poursuivre et punir.

Raisonnons paisiblement.

Il est reconnu que la loi est l'expression libre et solennelle de la volonté générale.

Voici le raisonnement qu'ont dû faire les partisans de la Constitution de 1793.

La Constitution de 1793 fut acceptée par 4800000 suffrages non compris ceux des armées qui ne furent pas consultées ${ }^{31}$.

Elle fut acceptée librement, car qui aurait pu forcer un si grand nombre de volontés : elle fut acceptée libremenent car à l'époque de cette acceptation il n'y avait en France ni législation des suspects, ni terreur, ni échaffauds, ni [p. 40] Bastilles.

Elle fut acceptée solennellement car 8000 députés vinrent apporter à Paris le voeu d'autant d'assemblées primaires, et assister au recensement par lequel il ne s'est élevé aucun doute.

Elle était bien certainement alors l'expression de la volonté du Peuple Français.

Il est survenu ensuite une nouvelle Constitution : tout homme raisonnable, tout bon citoyen a dû se demander : ce nouvel acte contient-il une nouvelle volonté du Peuple français dérogeant à celle qu'il avait émise en 1793.

(31) Buonarroti reprend, comme le font les Égaux, y compris pendant le procès, une évaluation fantaisiste publiée par Babeuf dans le numéro 34 du Tribun du peuple. Babeuf, comme presque tous ses contemporains, semble avoir ignoré le chiffrage des votes de 1793, quelques deux millions, mais dont le Comité de salut public avait fait renfermer le décompte dans l'Arche d'alliance. 
Il a pu considérer premièrement que d'après la Constitution de 1793 la Convention n'avait pas pu en proposer au Peuple une nouvelle.

Il a pu considérer que lors de l'adhésion à l'acte de 1795 il n'y a eu que 900000 suffrages pour déterminer la volonté générale.

Il a pu considérer que les voix des [p. 41] armées ont aussi été recueillies.

Il a pu considérer que le recensement fut fait d'une manière obscure et bien différente de celle qui fut pratiquée en 1793.

Il a pu considérer qu'en 1795 un grand nombre de citoyens languissaient dans les Bastilles, qu'un grand nombre était exposé aux poignards, et qu'un grand nombre fut expulsé des Assemblées primaires.

Et toutes ces considérations ont pu le déterminer très impérieusement à penser qu'en 1795 il n'y avait pas eu de nouvelle émission de la volonté du Peuple dérogeant à la première.

Et cela paraitra d'autant plus raisonnable au citoyen Viellart qui luimême n'a pu se dispenser de qualifier de fausse l'acceptation des fameux décrets des 5 et 13 Fructidor $^{32}$, quand il considéra que le recensement des suffrages pour l'acceptation de l'acte constitutionnel fut fait avec les mêmes formalités et en même temps que celui pour [p. 42] les deux décrets qu'il ne considère pas avec raison comme sanctionnés par le Peuple.

Vous remarquerez à ce sujet, citoyens Jurés, que cette violation même dont l'accusateur national convient franchement était de nature à faire penser que l'autorité existante en Floréal dernier n'était pas absolument légitime.

Permettez-moi de pousser ma considération encore plus loin.

Il y a entre la Constitution de 1793 et celle de 1795 une différence très remarquable. Celle-ci veut que les lois reçoivent leur validité par le sur le concours des deux Conseils, l'autre voulait que les décrets du Corps législatif ne fussent considérés comme lois que dans le cas où dans un délai déterminé il n'y aurait pas eu réclamation de la part du dixième des assemblées primaires de la moitié des départements plus un, et en cas de réclamation [p. 43] elle prescrivait de consulter la Nation.

(32) Les Conventionnels adoptèrent à la veille du vote populaire les décrets dits des deux-tiers qui permettaient, conformément au régime prévu pour le futur Directoire, de n'élire qu'un tiers de nouveaux députés dans les nouveaux conseils et donc de s'y conserver à hauteur des deux tiers. Ces décrets, mal diffusés, rarement soumis au vote furent, lorsque c'est le cas, souvent rejetés. La fraude ouverte qui aboutit ensuite à proclamer leur « adoption » est inséparable de la tentative insurrectionnelle de vendémiaire et pèsera toujours sur le Directoire. 
Vous voyez donc bien clairement que cette Constitution de 1793 garantissait au Peuple entier, [passage ajouté] paisiblement assemblé le droit de délibérer sur les lois, de les accepter ou de les refuser.

Or c'est ce droit qui peut avoir fortement frappé la tête de ceux qui, je suppose, voulurent rétablir cette constitution.

Ils avaient probablement lu dans Rousseau et dans Mably que sans ce droit la Souveraineté du Peuple disparait, la Nation n'est plus, la Loi n'est pas telle.

Ils avaient vu ce droit implicitement ou explicitement consacré dans toutes nos Déclarations des Droits.

Ils avaient probablement vu les arguments par lesquels les Philosophes anciens et modernes ont démontré que c'était dans ce droit que résidait [p. 44] le bonheur permanent de la Nation.

Ils avaient probablement vu dans l'histoire Romaine la bénédiction que l'introduction de ce droit accumula sur Valerius Publicola ${ }^{33}$.

[passage ajouté] Ils s'étaient probablement convaincus par la méditation et par l'étude que ce droit est naturel, inaliénable, inabdiquable. Ils avaient probablement pensé avec une multitude d'orateurs et d'écrivains que le recouvrement de ce droit de souveraineté fut le but de l'insurrection de 1789 et de toute la Révolution.

Et d'après toutes ces considérations fortes, pressantes, séduisantes ils ont pu raisonnablement penser que l'acceptation de la Constitution de 1795 avait été illusoire.

Y avait-il dans ce cas intention criminelle ? Et si de pareils hommes s'étaient trompés, ne faudrait-il pas reconnaitre dans leur détermination le désir du bien, la volonté de respecter l'autorité légitime, la prudence des recherches, la nécessité, l'impossibilité de se déterminer autrement, l'amour de la patrie, le zèle le plus constant et le plus illimité pour le service de la République ?

Deux objections ont été faites par [p. 45] l'accusateur national à ce raisonnement.

Il a prétendu d'abord que l'acceptation de 1793 fut aussi illusoire et arrachée par la terreur : je réponds par l'histoire.

Il a prétendu ensuite que mes arguments fussent-ils vrais les Jurés ne pourraient pas s'arrêter aux motifs qui ont pu déterminer les accusés,

(33) Un des fondateurs légendaires de la République romaine après la chute des derniers rois (509 av. Jésus-Christ). 
ni voir dans leurs intentions autre chose que l'intention de renverser la Constitution de 1795.

Je crois que si on admet cette étrange prétention il n'y a plus en France ni institution des Jurés, ni Patrie.

D'abord ce n'est pas sur le renversement de la Constitution actuelle mais sur celui de l'autorité légitime qu'il faudrait à mon avis porter l'attention des Jurés : car je ne puis pas me persuader qu'ils pussent déclarer coupable [p. 46] celui qui tout en agissant contre le Gouvernement actuel aurait fermement cru agir pour l'autorité légitime.

À quoi se réduirait alors ce sentiment intérieur du bien ou du mal qui rend si précieuse pour l'âme pure l'institution du Jury : à quoi se réduirait le soin que la Loi a pris de concilier par les questions sur l'intention et sur l'excuse les contradictions si fréquentes entre les préceptes de la Loi naturelle et ceux des Lois positives ?

À quoi se réduirait la Loi suprême de l'intérêt du Peuple qui ordonne à ses mandataires de compter pour principale circonstances dans le coeur des accusés l'amour de la Patrie et le dévouement pour elle?

À quoi se réduirait surtout cette bienfaisante règle de Philosophie et de Politique qui ordonne d'instruire les égarés et de ne punir que les méchants? [p. 47]

Je n'entretiendrai pas de nouveau les Jurés de la sagesse du Peuple Romain absolvant les défenseurs violents, et quelquefois indiscrets de la Souveraineté : vous êtes aussi Républicains : vous avez lu dans nos âmes : tout est légitime par la haine du vice et par l'amour de la Patrie.

Hâtez-vous, citoyens Jurés, hâtez-vous de prononcer sur mon sort, hâtez-vous de rendre au coeur d'une épouse tendre le calme que je m'efforce en vain de lui inspirer. Hâtez-vous de briser des fers qui réjouissent les Rois et leurs infâmes défenseurs.

Stéphanie RozA

Doctorante, Université Paris 1 - Panthéon Sorbonne CHSPM (Centre d'histoire des systèmes de pensée moderne) 7 rue Voltaire, 93400 Saint-Ouen stephanieroza@yahoo.fr 